\title{
The global challenge of cancer
}

\author{
Cancer is a multifaceted global health issue that continues to demand action. We are launching Nature Cancer to \\ inform, inspire and convene scientists across the multidisciplinary arena of cancer research, aiming to advance \\ biomedical knowledge and treatment strategies within the greater framework of society.
}

ancer is a disease that spans the breadth of human experience. Observed in hominid fossils and human mummies, first described in ancient times by Egyptian and later by Greek physicians, it has manifested itself throughout human history. Affecting people of all ages, cancer cuts through society, causing suffering on a global scale. According to the World Health Organization, cancer is responsible for one in six deaths, which makes it the second most common cause of death globally.

Through the ages, physicians who observed and described this disease were faced with its seeming intractability. The emergence of modern medicine changed that view through an initially slow accumulation of biological and therapeutic knowledge that accelerated with the advent of molecular cell biology and genetics in the latter part of the 20th century. This progress, together with more recent technological advances, have permitted an unprecedented understanding of the disease. Today the word 'cancer' refers to hundreds of distinct disease types that share similar fundamental properties. The importance of the tissue and cell type from which the disease originates is clear. It is known that the function of cancer cells at the molecular and metabolic level is crucial but is also highly context dependent. Cancer is also appreciated as a disease of change- $-\mathrm{a}$ condition characterized by plasticity and heterogeneity, that evolves at genetic, phenotypic and pathological levels, and progresses through different stages clinically. Beyond decoding of the genetic fingerprint and molecular makeup of a specific cancer type, we understand the importance of the systemic and local tumor environment in how the disease develops and manifests. The interplay with the immune system and immune tumor microenvironment has become especially apparent in recent years. Indeed, today we recognize that cancer heterogeneity, evolution and the local and systemic environment all have key roles not only in disease development but also in the response or resistance to therapy and disease recurrence.

Technological advances such as nextgeneration sequencing, integrated '-omics', imaging and single-cell methodologies have allowed profiling of different tumor types at a resolution and scale that were not possible previously. The ability to generate and share big data is fundamentally altering the way this disease is understood and treated-for example, by allowing the identification of biomarkers to select patients for clinical trials and evaluate therapy responses. Data science has become a core part of a field that is increasingly embracing computation, as in the form of artificial intelligence for extracting information from complex datasets. Nevertheless, the potential of such approaches to revolutionize data analysis for cancer screening, diagnosis and therapy decisions comes with challenges.

Viewing cancer as a systemic disease characterized by evolution, heterogeneity and environmental inputs may seem commonplace now, but in reality, revealing one layer of complexity only underscores other complex features that need to be appreciated. The size, quality and complexity of large datasets, such as those of The Cancer Genome Atlas, the International Cancer Genome Consortium and the Human Cell Atlas (among others), mean that considerable work is needed to decode, interpret and contextualize findings. Identifying tumor cell-intrinsic genomic and epigenomic attributes provides only a snapshot of tumor development and progression. A more complete picture may emerge with longitudinal information, as well as profiling of different cellular constituents of tumors, such as stromal and immune cells. Integrative '-omics' and single-cell approaches provide the ability to do so; however, additional factors need to be considered. Among them are the peculiarities of the particular tissue and tumor type, the size and characteristics of human-participant cohorts or the choice of preclinical animal model systems, the resolution and strength of the chosen methodology and the quality of analytical tools. How data from individual patients versus larger cohorts are handled and analyzed, the information that can be obtained from each type of analysis and the extent to which tumor profiling studies may be more broadly generalizable, given the degree of inter-patient heterogeneity, are questions with which this field continues to grapple.

The developments noted above have also revolutionized the approach to treatment. The more granular understanding of cancer's molecular drivers and tumor-cellintrinsic or extrinsic vulnerabilities, and the addition of next-generation sequencing testing to clinical practice, have given rise to targeted therapies and the concept of precision oncology - treatment tailored to the individual patient, aiming to hit cancerspecific vulnerabilities, thereby hopefully reducing toxicities and improving quality of life for patients receiving treatment. Highthroughput approaches, computational science, bioengineering and nanomedicine are changing the landscape of drug and diagnostics development. The hard-won advances in tumor immunology have led to an explosion of cancer immunotherapies. These therapeutic breakthroughs in precision medicine and immunooncology have successfully introduced several therapeutic modalities into the clinic. However, as with historical cancer treatments, these new modalities still encounter the setbacks of therapy resistance and lack of response, as well as their own serious adverse events. To that end, studying the fundamental mechanisms underlying these processes to devise new therapeutic strategies, and exploring innovative treatment combinations, represent major areas of focus.

An additional key consideration in the effort against cancer is the influence of the environment, daily habits and culture. We are gaining a better understanding of these facets of the disease, but such factors are often difficult to quantify and control in a real-world setting, or to model in the laboratory. The often late-stage presentation, and therefore late diagnosis, of the disease continues to hamper therapy options, and metastasis remains a major cause of cancer deaths and a main focus of foundational cancer research. Socioeconomic factors lend an additional, devastating dimension: according to the World Health Organization, $70 \%$ of cancer deaths occur in low- or middle-income countries, but even in high-income societies, certain parts of the population bear a disproportionate burden 
of suffering. A large fraction of cancer cases and deaths may be preventable with greater epidemiological and mechanistic understanding of environmental and behavioral risk factors. The development and wider adoption of the Pap test and HPV vaccines against cervical cancer are singular successes that exemplify the importance of early detection and prevention in neutralizing the threat of cancer in a broad population.

However, this remains a disease of disparities. Therefore, it is essential to deepen our appreciation of the underlying causes of these inequalities and to work toward reversing them, always keeping the patient at the forefront of the cross-disciplinary scientific endeavor in this field. Developing moreeffective screening and diagnostic means and working toward providing accessible and affordable high-quality cancer care for the wider population will be essential for addressing cancer-health disparities.
It has been thousands of years since the ancient Greek physician Hippocrates strove to understand this disease and named it 'cancer' from the Greek word karkinos, meaning 'crab', possibly as an allusion to the blood vessels emanating from tumors. We are now in the enviable position of having a much more refined understanding of the disease and we know that cancer has no panacea. Instead, it requires synthesis of knowledge, collaboration between fields and a deeper appreciation of the challenges facing patients, clinicians and scientists from different disciplines. Fortunately, this is an era of thriving biomedical research that has seen the field of cancer research expand into a vibrant, multidisciplinary community that seeks new and innovative ways to engage collectively and to tackle this disease.

As we launch Nature Cancer, we seek to provide a unique forum that embraces the breadth of this community, from foundational preclinical science to translational and clinical work. Through our pages, we aim to increase the knowledge of cancer formation, development and progression, to explore innovative approaches to cancer diagnosis, treatment and prevention, and to understand the societal impact of this disease. Ultimately, our goal is to become a point of convergence for scientists from diverse fields and to lend a new voice to discussing and contextualizing the most exciting findings and pressing issues in cancer research today. Our inaugural issue encapsulates the diversity of science we aim to bring to you and the conversations we seek to start. As we realize our first steps into this field, we thank our authors and referees and welcome our readers.

Published online: 13 January 2020 https://doi.org/10.1038/s43018-019-0023-9 